A third point which may have some effect on character is that the major part of eye work is meticulous and of an almost mathematical exactness which predisposes to clear thinking.

There are many other features of ophthalmic work which have been omitted in this short review, for example, the rush and hurry of a crowded out-patient department, the attempt to do good work with inadequate equipment, the realisation when teaching undergraduate students that ophthalmology is only a small branch of medicine and that all they want is the irreducible minimum of information. The first two may induce a feeling of frustration, but the last may beget some humility and a realisation that great though the field of ophthalmology may be, it is only part of the vast acreage of medicine, much of which we have not explored.

Such then is the environment in which we live and it seems to produce a kindly race of men with a wide knowledge of humanity, an ability to come to quick and wise decisions and a flair for accurate observation. That these and other qualities must have been present in some degree before engaging on ophthalmology is, of course, obvious, but that the practice of our profession encourages their growth seems a likely possibility.

\title{
ANGIOMATOSIS RETINAE, WITH REPORT OF PATHOLOGICAL EXAMINATION
}

BY

\section{HUMPHREY NEAME}

LONDON

IN view of (1) the rarity of the condition (2) the lack of reports of late results of treatment, and (3) the fact that the affected eye of the second case was excised and examined pathologically, the following two cases seem worthy of record. One, Ada C., was first examined in 1936, had both eyes affected and had varied treatment; the second, Violet H., was seen at Moorfields by several surgeons and a fundus drawing was made in 1939; the writer did not see her until November, 1946, when the affected eye was found to be painful, blind and glaucomatous, and was therefore excised.

Case 1. Ada C., aged 28 years, attended Moorfields Eye Hospital on February 8, 1936, complaining of blurring of the sight of the left eye, apparently of sudden onset.' At that date the R.V. was $6 / 66 / 5$ (partly), the L.V.6/60. After dilatation of the pupils, examination of the right fundus through clear media showed a disc of healthy appearance, two very dilated vessels passing into the 
inferior temporal region, and some hard white exudate like that of circinate retinopathy mostly between the vessels (see Fig. 1). In the 7 o'clock direction a rounded prominence of pinkish colour was visible in which the two large vessels were lost. The left eye, whose

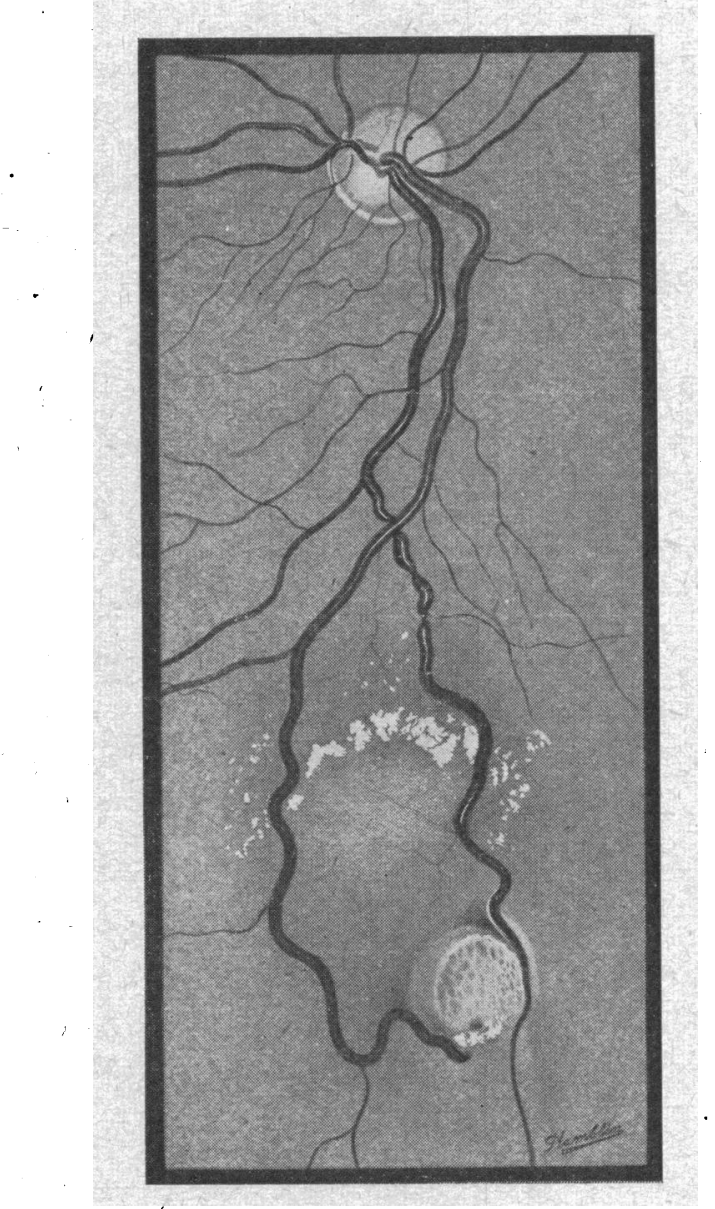

Fig. 1.

Ada C. Fundus drawing of the right eye, showing two dilated retinal vessels ending in a pinkish-coloured localised prominence. (The drawing has been rotated to save space. The angioma was in the 7 to 7.30 o'clock meridian).

media were also clear, showed two vessels of very large calibre passing from the optic disc in an upward direction, the larger being also very tortuous (see Fig. 2). A large area of hard white exudate occupied most of the area from the optic disc to the macula. Small retinal vessels lay anterior to the exudate. The enlarged 


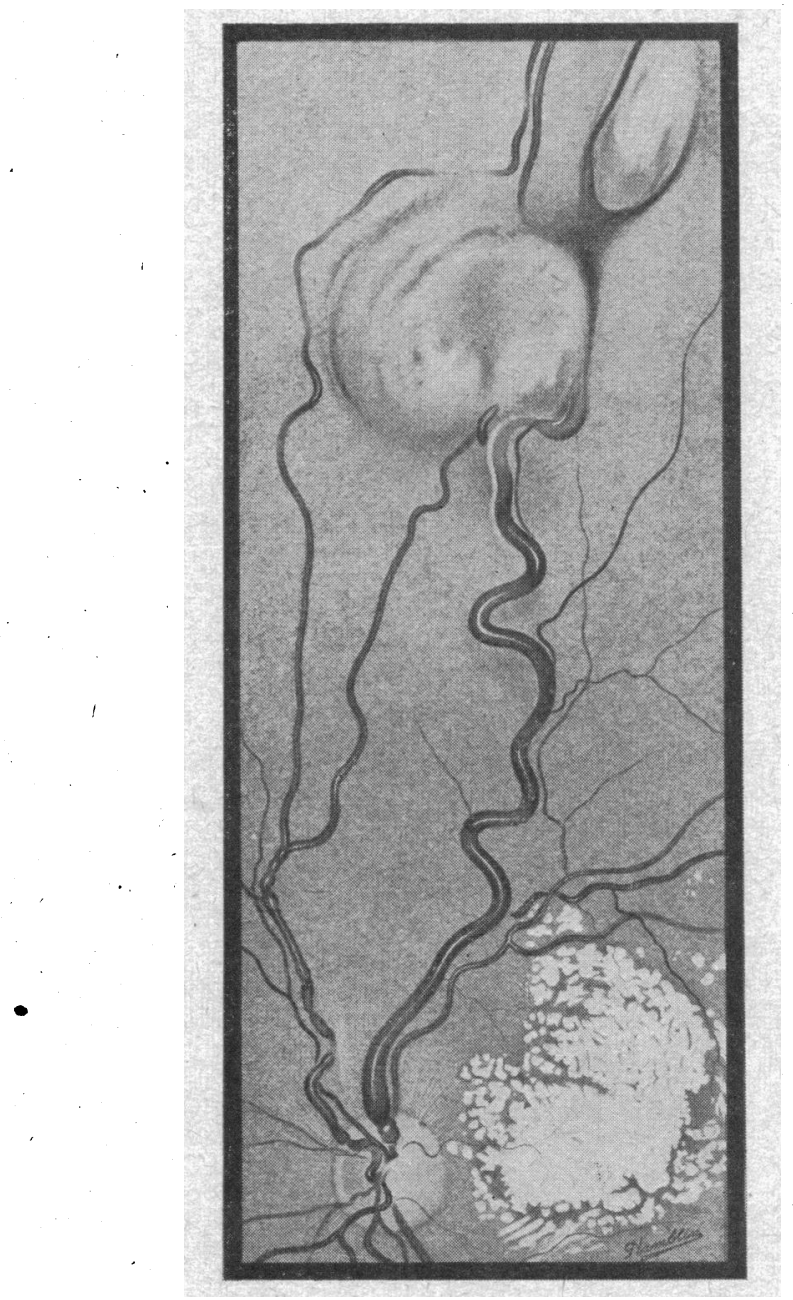

Fig. 2.

Ada C. Fundus drawing of the left eye, showing a similar appearance to that of fig. 1, but with two swellings in the upper periphery. .

blood vessels led up to an oval pinkish prominence, in the 12 o'clock direction, fully 2 disc diameters in vertical dimension and rather less transversely. About 1 disc diameter further and slightly to the temporal side lay another similar prominence of smaller size. The retina in the region of the swellings and of the enlarged vessels had the appearance of being slightly detached.

By estimating disc diameters both from the disc to the swellings and from the swellings to the extreme periphery and assuming the 
distance from disc margin to the limbus to be about $36.5 \mathrm{~mm}$. in the two directions, it was calculated that the angioma in the right eye was $18.5 \mathrm{~mm}$. from the limbus; in the left it was judged that the anterior limit of the smaller angioma was $23 \mathrm{~mm}$. from the limbus.

Treatment of the left eye. Between February and June, 1936, the left eye was treated by electrolysis with a fine perforating needle 3 times. A galvanic current by the cathode was used at 2 milliamperes for 4 seconds to 4 milliamperes for 6 seconds, 10 to 12 punctures at each operation. A further application of electrolysis, 5 milliamperes for 10 seconds at each of 27 perforations, was made in June, 1937. Only at the second application of the $3 \mathrm{~mm}$. fine needle did well-marked bubbles appear in the vitreous. At the end of this operation the whole of the angioma area was surrounded by the pale reaction of electrolysis. The 2 large retinal vessels were much reduced in calibre and little larger than normal retinal vessels.

As during succeeding months of 1937 the 2 angiomata in the left eye showed no signs of regression, treatment by the 1 gram radium bomb was applied. It is regretted that details of dosage cannot be traced. The patient, however, declares (1948) that 8 separate hours of treatment were given and further treatment stopped as loss of eyelashes took place in the lower eyelid. A total dosage of 14 hours had been planned. In the latter part of 1938 no definite improvement in the condition was detected. It was therefore decided to employ diathermy with a perforating needle.

On December 7, 1938, perforating diathermy was employed with $\tau$ punctures. One of these coincided with the larger retinal vessel and one with the angioma. At the end of the operation the vitreous was very dark and vision barely perception of hand movements.

On January 2,1939 , the L.E. had vision still no more than perception of hand movements. The vitreous was very dark with a faint red reflex above.

Treatment of the Right Eye. On September 12, 1936, the right eye was treated by electrolysis under local anaesthesia aided by luminal and omnopon. A guide suture inserted at the limbus was carried across the cornea in the 6.30 meridian. A small puncture was made $22 \mathrm{~mm}$. from the limbus in this line with a broad needle and a Foster Moore stud inserted. 'This showed ophthalmoscopically'a white streak immediately posterior to the angioma and slightly temporal.

A galvanic current was then applied by means of a $3 \mathrm{~mm}$. sharp needle on an insulated curved shaft, connected with the cathode. A current of 10 milliamperes was used at 12 points for 6 seconds 
each. The punctures were made as far as possible in a square area, at 1 to $1.5 \mathrm{~mm}$. apart just anterior to the stud mark. At the end of the operation a mass of small bubbles was seen with the ophthalmoscope just posterior to the angioma and a wide area of pallor anterior to these. Finally 2 or 3 more punctures were made posterior to the stud in the hope of destroying the enlarged vessels.

On October 5, 1936, the vitreous was hazy but a large area of reaction was visible below with an outlying patch just below the macular area (from one of the latest applications).

On November 11, 1936, the R.V. with $+2 \cdot 25 \mathrm{sph} .=6 / 12$ the pupil being dilated. L.V. with $+1 \cdot 25=6 / 24$. The angioma was visible in the right eye in an area of atrophy, but was estimated to be $2 / 3$ disc diameter as compared with the estimate on September 10, 1936, of $1 \mathrm{~d} . \mathrm{d}$. across.

Progress. On May 18, 1939, two weeks after a blow on the forehead the patient noticed a cloud in front of the right eye. Several streaks of haemorrhage were visible on this date in the right vitreous. Dark streaks were still visible in the lower part of the vitreous cavity in March, 1940. The appearance of slight retinal detachment is noted below. Posterior cortical lens opacities are seen in the left eye, and a large retinal cyst in the lower periphery.

September 10,1940 . R.V. with lens $=6 / 9$, L.V. with lens less than $6 / 60$. In the right, white proliferating tissue extends upwards from the most posterior point of electrolysis towards the fovea. Increasing retinal detachment below : L.E. anterior and posterior iridescent cortical lens opacities.

January 2, 1941. R.E. a delicate veil from the disc passes towards the macula. The lower retina is quite definitely detached. L. disc is barely visible owing to lens opacities.

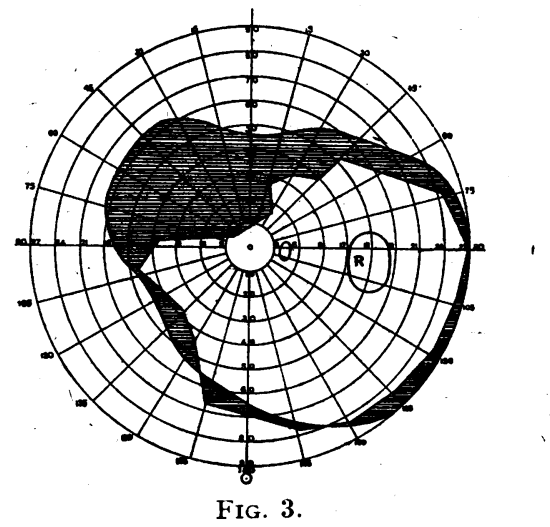

Ada C. Field of vision of right eye on October $22,1941 . \frac{1}{2}^{\circ}$ white object. 
Field of Vision. Fields mapped by the perimeter before operation with $1 / 4^{\circ}$ white object were full. The chart of October 22, 1941, with $1 / 2^{\circ}$ white shows a representative right field of several charted after operation (Fig. 3). Bjerrum screen indicated encroachment towards the macula in the upper nasal region to the $8^{\circ}$ circle (4/2000 white object) on November 11,1936 .

On July 8,1942 , the loss had extended to the $3^{\circ}$ circle $(6 / 2000$ white), and on January 20,1948 , to the fixation point $(5 / 2000$ white).

Visual acuity. November 11, 1936. R.V. (pupil dilated) with $+2 \cdot 25 \mathrm{sph} .=6 / 12$, L.V. $+1 \cdot 25=6 / 24$.

In 1943 on several occasions $R . V$. recorded as $6 / 9$ with glass. In December, $1944, \mathrm{R} . \mathrm{V}$. with glass $=6 / 18$, and complaint made of recent dimness of sight. This was explained by vitreous floating opacities of a reddish colour.

November 6, 1946. R.V. with glass 6/12, 6/9 (4).

$$
\text { January 20, 1948. (H.N.) R.V. } \frac{+2.5}{+0.25 \text { ax. } 90^{\circ}}=6 / 246 / 18 \text { (3). }
$$

L.V. perception of light with faulty projection.

\section{Discussion}

In view (1) of the tendency to bleed into the vitreous, as also observed by Foster Moore ${ }^{1}$ after radon treatment, (2) of the very slow absorption of blood, and (3) the rucking of the retina which is evident between the disc and the lower part of the right macula and hence a fear of proliferating retinopathy, no further operative treatment to the right eye has been thought advisable.

In view of the slowly progressive loss of field with increasing retinal detachment below, it is intended to recommend diathermy puncture in 2 places in the hope that retinal cyst formation is the cause of detachment as occurred in the left eye.

Case 2. Violet H., married, aged 20 years. The patient attended Moorfields Eye Hospital early in October, 1946, complaining that the left eye had been aching on and off for two months.

Previous History. She had been to the London Hospital in 1935 where it was stated that she had a " birth-mark" at the back of the left eye.

In October, 1939, she first visited Moorfields. The writer first saw her in 1946. The recent in-patient notes record that she had been examined in 1939 by several members of the Honorary Staff and that the diagnosis of angiomatosis retinae was made. (See Fig. 4, drawing of October 5, 1939.) She was admitted on November 5,1946 . The right eye was free from congestion, the 


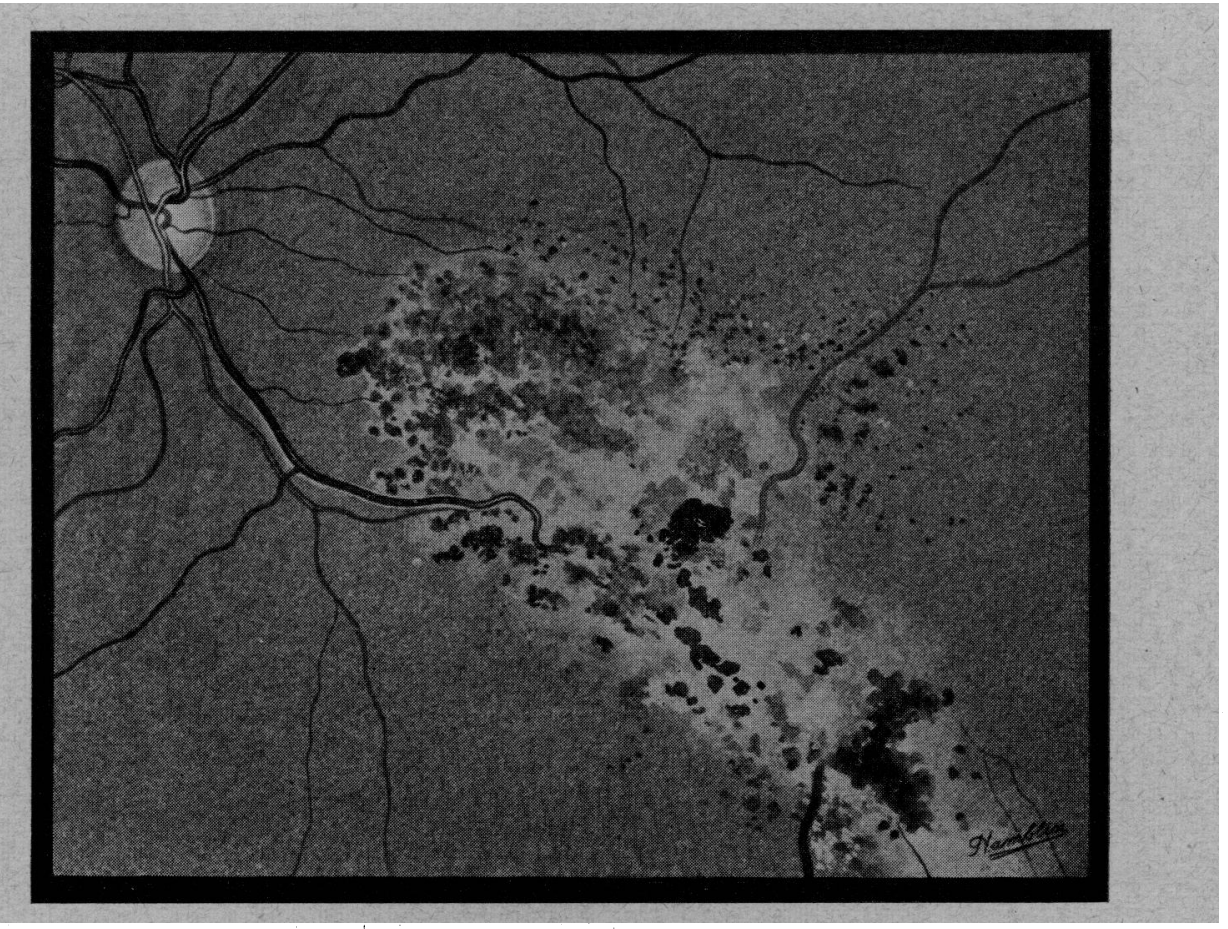

Fig. 4.

Violet H. Fundus drawing, left eye, October 5, 1939.

pupil circular and active, the media were clear and the fundus healthy. With -4.5 dioptre sph. -0.5 dioptre cyl., $180^{\circ}$, vision was 6/6. Tension normal. The left eye was white, the cornea clear, and the pupil circular with no direct reaction to light and sluggish consensual reaction. No red reflex was obtained. A glistening mass was seen close behind the lens with numerous straight vessels coursing over it. The tension was much raised and there was no perception of light. To transillumination there was relative dullness below. The left eye was excised on November 6, 1946.

Pathological examination. The eye, fixed in Zenker's fluid, was embedded in celloidin and antero-posterior sections were cut. These were stained with haematoxylin and eosin.

Histological Examination. Path. No. R.L.O.H. 271/1946.

Antero-posterior sections examined under Zeiss A. objective and No. 4 eye-piece showed a shallow anterior chamber whose angle was closed by apposition of the iris root to the cornea, completely 
covering the region of the canal of Schlemm. There was atrophy of the iris and ciliary body with slight ectropion of the uvea. The lens was in situ and had retina closely in apposition to its posterior surface. The vitreous cavity was almost entirely obliterated by complete retinal detachment. A few small round cells and fibroblasts

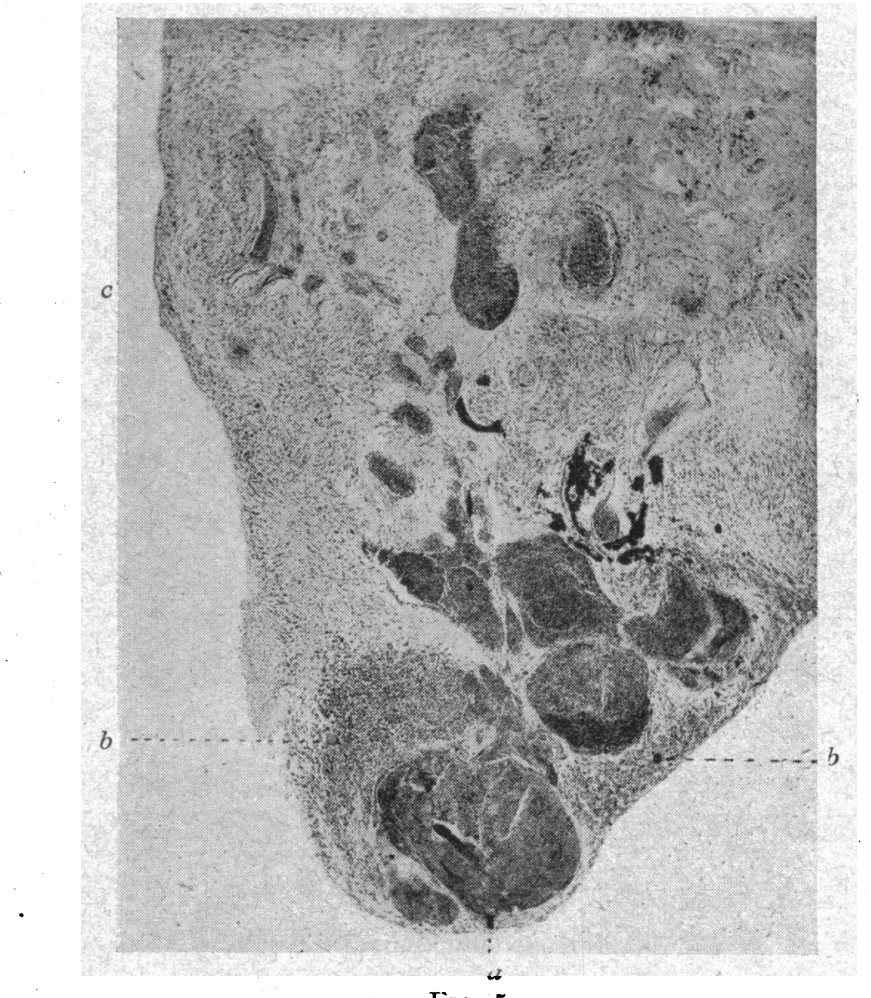

FIG. 5.

Violet H. Microphotograph ; magnification $\times 35$; shows the most posterior part of the angiomatosis area of the detached retina. (Stained with haematoxylin and eosin). (a) indicates the largest blood-vessel in the group. Abundance of coarse vessels extend forwards in the cone-shaped mass of detached retina to an additional extent about equal to that shown in this photograph. (b) marks areas of haemorrhage in the retina.

were near the reflexion of the retina at the ora serrata. In the posterior folded up part of the detached retina were blood-vessels of large calibre with a number of smaller vessels lying among retinal elements whose layers cannot here be indentified. Partly surrounding several of the large vessels were masses of red corpuscles - haemorrhages, which may have been responsible for the recent subacute glaucoma (Fig. 5 , microphoto 1 ). There was no trace of 
any wall or boundary to indicate any localisation of the vascular growth. In fact an abundance of vessels extended in the section for about twice the expanse shown in Fig. 5, i.e., some 6 millimetres in all. The subretinal space was partly occupied by some granular exudate containing numerous migrated rounded pigment cells with circular nuclei. The choroid vessels were full of red blood corpuscles and a fairly widespread round-celled infiltration affected the spaces between the vessels.

Examination under higher magnification showed the large bloodvessels to be very thin-walled, having little more than a single layer of endothelium. The layers of the retinal structure could only be identified in the periphery, near the ora serrata. (Fig. 6, 7, 8.) No cystic changes were present in the sections examined.

The striking feature of this angioma is its diffuseness and complete absence of any definite boundary. Its extent in the sections shows that it had enlarged somewhat in the 7 years since the drawing was made. There is, however, no suggestion in the sections of malignancy. A comparison with the reproductions of the microphotographs of McDonald and Lippincott ${ }^{2}$ shows a very close resemblance in structure. These writers, using glial stains, found only slight proliferation of the glial cells and concluded that this was secondary.

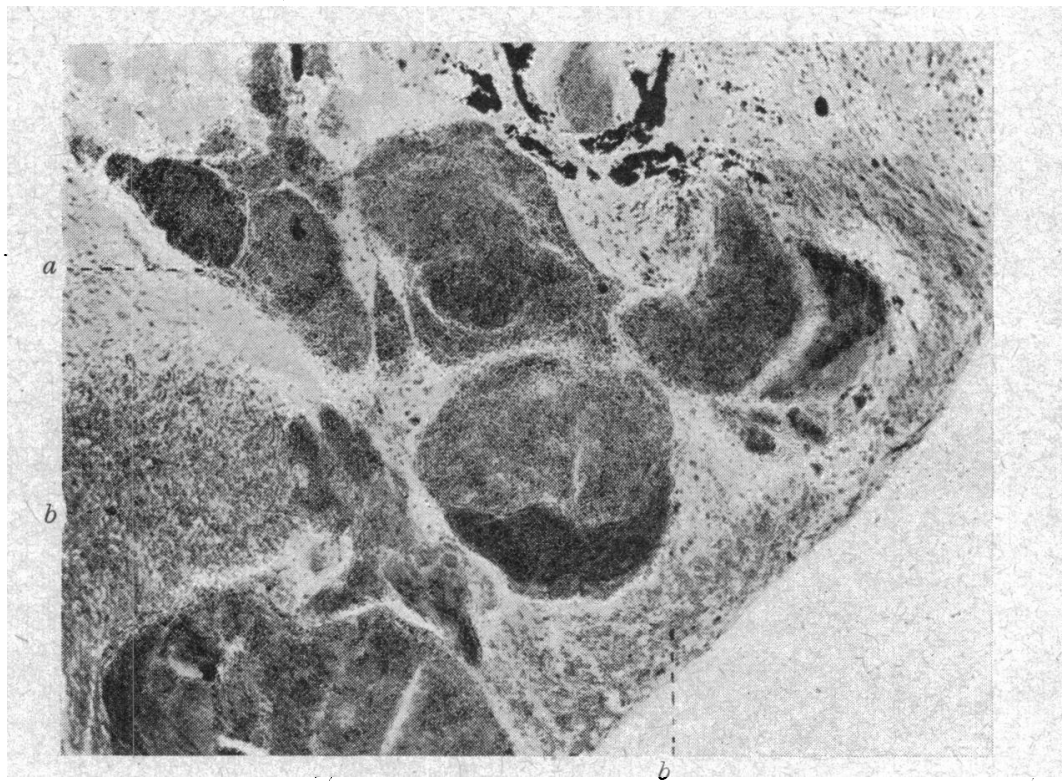

FIG. 6.

Violet H. Microphotograph; magnification $\times 80$. Portion of the section shown in Fig. 5 . For: $(a)$ see Fig. 7 ; $(b)$ haemorrhage. 


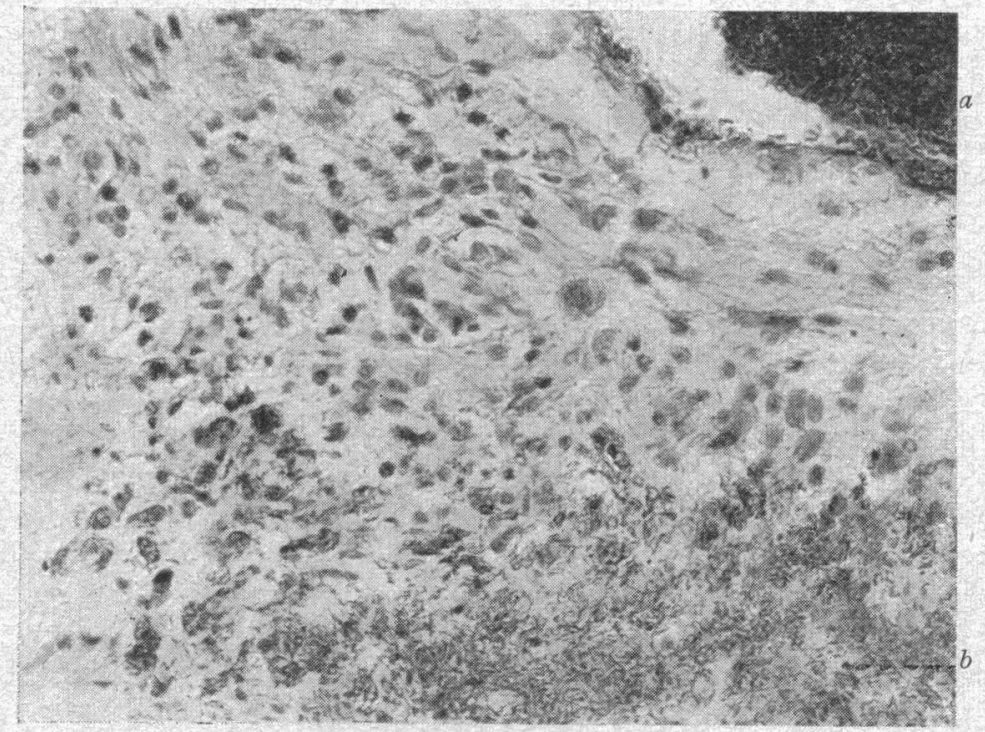

Fig. 7.

Violet $\mathrm{H}$. Microphotograph ; $\times 260$. The portion shown corresponds with $(a)$ in Fig. 6 ; $(b)$ marks the edge of a haemorrhagic area in the detached retina.' The blood has shrunk away from the vessel wall which shows a single layer of endothelium.

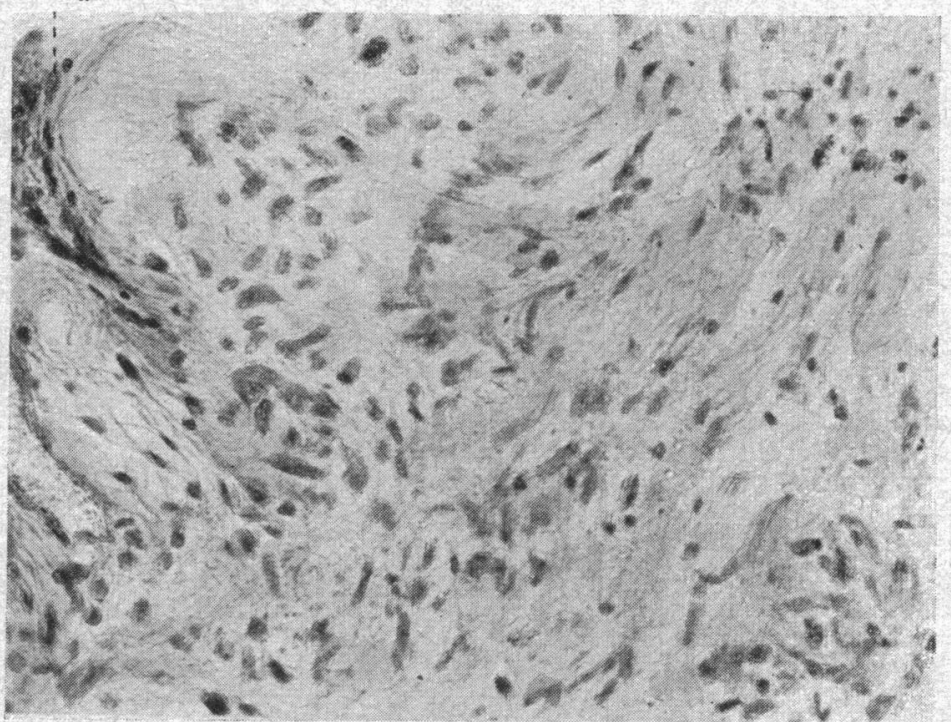

FIG. 8.

Violet H. $\times 260$. Showing cellular structure. $(a)$ indicates a flattened blood-vessel which can be recognised opposite $(c)$ in Fig. 5. (This vessel is at right-angles to its direction in Fig. 5. The lower border of Fig. 8 corresponds with the left-hand border of Fig. 5). 
Nicolotte D. M. H. Whiting's case of bluish juxta-papillary angioma. October, 1945.

FIG. 9. 
In addition to the above the writer has had the opportunity of examining two cases of a bluish-coloured angioma of venous appearance in addition to one reported by Nicoll and Moore ${ }^{3}$. The latter is also reproduced by Elwyn ${ }^{4}$. The two cases were seen at Moorfields Eye Hospital in the clinics of Mr. M. H. Whiting and Mr. E. F. King to whom the writer is indebted for permission to refer to them. They were of exactly the same type as Nicoll and Moore's seen in 1934. In all three the bluish coloured prominence with a nodular surface was at, or overhanging the optic disc.

Case 3. Nicolette D. (Fig. 9). Aged 7 years. The patient was under Mr. Whiting at Moorfields Eye Hospital Out-Patient Department on September 26, 1945. R.V.6/9. L.V.6/6. A mulberry-like growth was seen to overhang the disc margin of the left eye. This had been noted by Mr. Joshua Keyms of Southampton who referred the case to Moorfields. The purplishcoloured mass was approximately circular and extended about half way over the disc. It was nodular, and definitely prominent. It appeared to be composed mainly of rather large veins (larger than the ordinary tributaries of the central vein).

A follow-up has recently been possible, thanks to the help of Mr. W. E. Heath of Rochester, in the first of two cases of capillary angioma treated by Moore ${ }^{1}$ in 1934 . The patient stated that her sight remained good for nearly 18 months after the radon treatment and then failed fairly rapidly owing to cataract.

Types of Angiomatosis Retinae. There seem to be two distinct clinical types; (1) the pale pinkish swelling or swellings connected with retinal vessels of fully 2 to 3 times the ordinary calibre. These are usually circumscribed and appear clinically to have an enclosing sheath or capsule. Rarely they appear to be without any capsule-see drawing of case 2 (Violet H., Fig. 4), as is confirmed in the histological sections. In these the prognosis is bad without treatment; (2) the bluish coloured venous-looking swelling of which three cases have been seen. One of these (Nicoll and Moore ${ }^{3}$ ) was excised and found to be non-malignant. These cases should be followed up to see if the swellings increase in size. Elwyn associates cases of other types of vascular anomaly with these; (1) Coats' disease with telangiectases, (2) Sturge-Weber disease, occasionally with dilated and tortuous retinal vessels; and (3) the extremely rare arterio-venous aneurysm of the retina.

TREATMENT. The pinkish capillary angioma associated with 2 vessels of large calibre, must be treated, as it leads inevitably to blindness. Radium, radon and X-rays have been used. In all of these cataract is to be expected as in Moore's case (radon ${ }^{1}$ ) sent by Heath, and the left eye of the author's case No. 1. In the 
latter, to the left eye with two angiomata-one as much as $3 \mathrm{~mm}$. across, with localised detachment already present-electrolysis applied on three separate occasions between February and June, 1936, did not suffice. Radium bomb was then employed in 1937. However, no notable change in the condition followed. Perforating diathermy was employed in December, 1938, as described, with vitreous haemorrhage. Cystic degeneration of the retina and cortical cataract were present 2 years later. The angioma in the right eye appeared to be swallowed up in fibrous tissue after electrolysis, but 2 bouts of vitreous haemorrhage have occurred since and a lowly progressive detachment of the retina. Foster Moore's second case treated by radon was not traceable. Of two cases treated by diathermy Weve' $s^{5}$ had vision $1 / 62 \frac{1}{2}$ years after treatment of an angioma in the superior-temporal region above the macula, and retained practically a normal field. Michaelson' ${ }^{6}$ case with an angioma not far to the temporal side of the macula and with a localised retinal detachment and a retinal hole, recovered to the extent of vision $6 / 9$ with correction as compared with $6 / 36$ before operation. This, however, was only, $3 \frac{1}{2}$ months after operation and the angioma was only $1 / 3$ disc diameter across.

The author favoured the use of the galvanic current as this has been a favourite method of treating spider-naevi in the skin of the face. It was hoped to puncture the angioma and apply sufficient current by the cathode to cause haemostasis. It is probable that with any but the smallest angiomata-e.g., Michaelson's -this is still the ideal method. But the method of approach may perhaps be improved. It may be mentioned that the electrocautery is used by some dermatologists to destroy a spider naevus. As in the eye, however, only one shot can be made by this instrument 'and a bull's-eye must be scored, this technique is not feasible for the retina. It seems that, provided the angioma is not far in the periphery, a fine but rigid needle insulated nearly to its point might be used by the trans-scleral route while the fundus is under observation with the binocular ophthalmoscope through Goldmann's contact lens ${ }^{7}$. The latter used this method to apply diathermy to a macular hole in order to prevent detachment of the retina, as described by Foster ${ }^{8}$. In the second type of angioma, that composed of coarser bluish vessels, of which three cases have been mentioned, should definite evidence of increase in size be obtained, this may prove to be the best method of attack: The help of a physicist is needed in order to choose between the merits of galvanism or diathermy. In the 3 cases mentioned it would probably be desirable to use the kind of current whose destructive effect is the less extensive, so as to cause as little damage 
to the optic nerve as possible. In any case, there is a great probability that almost half the visual field would be lost. When the angioma is to the temporal side of the optic disc-macular vision would be destroyed. Better that, however, than loss of the eye.

Several unsolved problems exist in connexion with this condition :-

(1) Is there a tendency for new angiomata to form as occurred in one case mentioned and illustrated by Elwyn ${ }^{4}$ ?

(2) Hard white exudates may be present early as in the right eye of case 1 described, long before any detachment was suspected. Are these due to haemorrhage in one whose retinal vessels are especially prone to leak?

(3) Why does retinal detachment occur when there is apparently little subretinal exudate and no hole in the retina?

\title{
BIBLIOGRAPHY
}

1. Moore. R. F,-Trans. Ophthal. Soc. U.K., Vol. LV, p: 3, 1935, Proc. Roy. Soc. Med., Vol. XXVI, p. 1036, 1932.

2. McDonald, R. and LippincotT, S. W.-Arch. of Ophthal., Vol. XX, p. 958, 1938.

3. Nicoll and Moore.-Brit. Jl. Ophthal., Vol. XVIII, p. 454, 1934.

4. ELWYN, H.-Diseases of the Retina, London, pp. 169, 177, 1948.

5. Weve, H.-Trans. Ophthal. Soc. U.K., Vol. LIX, p. 43, 1939.

6. Michaelson, I. C.-Brit. Jl. Ophthal., Vol. XXVIII, p. 522, 1944.

7. GoldmanN, H.-Ophthalmologica, Vol. XCVI, p. 90, 1938.

8. Foster, J.-Brit. Jl. Ophthal., Vol. XXXII, p. 83, 1948.

\section{VISUAL PROTECTION IN AERIAL WARFARE}

\author{
Air-Marshal P. C. Livingston
}

\section{INTRODUCTION.}

IN order that men may bear weapons with success and fortitude in modern warfare, it is necessary that they be armed, not only with equipment suitable to the occasion, but with their minds prepared for such high adventure that the capacity for imagination is locked within the lower levels of consciousness. Throughout the World War, 1939/45, personal experience records no case where a member of aircrew became obsessed by a dread of blindness through enemy action. On the contrary, the destruction of sight appeared so little feared, that difficulty was experienced in persuading personnel of the air arm to take reasonable precautions against ocular injury. This negation of the thought of blindness 